\title{
Universality and Anomalous Mean-Field Breakdown of Symmetry-Breaking Transitions in A Coupled Two-Component Condensate
}

\author{
Chaohong Lee* \\ Nonlinear Physics Centre and ARC Centre of Excellence for Quantum-Atom Optics, \\ Research School of Physical Sciences and Engineering, \\ Australian National University, Canberra ACT 0200, Australia
}

(Dated: October 26, 2018)

\begin{abstract}
We study both mean-field and full quantum dynamics of symmetry-breaking transitions (SBTs) in a coupled two-component Bose-Einstein condensate. By controlling s-wave scattering lengths and coupling strength, it is possible to stimulate SBTs between normal and spontaneously polarized ground states. In static transitions, the probability maxima of full quantum ground states correspond to the mean-field ground states. In dynamical transitions, due to the vanishing of excitation gaps, the mean-field dynamics shows universal scalings obeying Kibble-Zurek mechanism. Both meanfield and full quantum defect modes appear as damped oscillations, but they appear at different critical points and undergo different oscillation regimes. The anomalous breakdown of mean-field dynamics induced by SBTs depends on the approaching direction.
\end{abstract}

PACS numbers: 03.75.Kk, 03.75.Mn, 64.60.Ht, 05.30.Jp

In a many-body quantum system, spontaneous symmetry breaking (SSB) occurs if its mean-field (MF) states do not possess symmetry of its original many-body Hamiltonian. Since atomic Bose-Einstein condensates (BECs) have long collision times, they are excellent candidates for testing intrinsic mechanisms of the SSB [1]. In particular, the simultaneous realization of superfluidity and magnetism (or spin polarization) in spinor BECs is associated with the SSB related to both external and internal degrees of freedom. For spin-1 BECs, the experimental observation of SSB [2] has triggered several theoretical studies [3, 4]. For spin- $\frac{1}{2}$ (two-component) BECs, the phase separation has been observed experimentally [5], and their spatial SSB [6] and spontaneous spin polarization [7] have been predicted theoretically.

It is well known that, in slow processes, the gapped excitations over zero-temperature ground states obey Landau-Zener mechanisms. However, gapless excitations appear in almost all systems with SSB. Because of the gapless excitations, the adiabaticity breaks down [8] and the generated defect modes [3, 4, 9] follow KibbleZurek (KZ) mechanisms [10]. The current studies of dynamical mechanisms for SBTs focus on lattice systems [8, 9, 11, 12], spin-1 BECs [3, 4] and other manybody systems by employing either MF [4, 11] or full quantum (FQ) [3, 8, 9, 12] theories. However, (i) few works compare the MF and $\mathrm{FQ}$ dynamical mechanisms near a critical point to explore regimes of correspondence, and (ii) the dynamical mechanisms for SBTs in two-component BECs are still not clearly understood.

In this Letter, we analyze both MF and FQ dynamical mechanisms for SBTs in a coupled two-component BEC. For simplicity, we only consider the SSB related to internal degrees of freedom, i.e., two hyperfine spin states. In static transitions, the MF ground states correspond to the probability maxima of the $\mathrm{FQ}$ ground states. If the coupling strength increases from zero to a sufficiently large quantity, the MF ground states transfer from being spontaneously polarized (self-trapped) to non-polarized (normal). Correspondingly, the two lowest FQ eigenstates transfer from being quasi-degenerated to non-degenerated. In dynamical transitions, due to the disappearance of gaped Bogoliubov excitations, the MF dynamics obeys an universal KZ mechanism and the defect modes are induced by dynamical instability. Due to the non-identity of the $\mathrm{FQ}$ and MF critical points, the MF breakdown induced by SBTs depends on the approaching direction. The dynamical mechanism of SBTs connects with the quantum adiabaticity, which provides various applications in atomic physics, condensed matter physics and non-equilibrium dynamics, and particularly in adiabatic quantum computation [13].

We consider a gaseous BEC of bosonic atoms which populate two hyperfine levels coupled by external fields. Assuming that the coupling fields are spatial uniform and the atom-atom interactions do not change the internal states, the system obeys a second quantized Hamiltonian [14],

$$
\begin{aligned}
H(t) & =H_{0}+H_{i n t}+H_{c}(t), \\
H_{0} & =\sum_{j=\uparrow, \downarrow} \int \hat{\Psi}_{j}^{+}(\vec{r})\left(-\frac{\hbar^{2} \nabla^{2}}{2 m}+V+\epsilon_{j}\right) \hat{\Psi}_{j}(\vec{r}) d^{3} \vec{r}, \\
H_{i n t} & =\frac{1}{2} \sum_{j, k=\uparrow, \downarrow} U_{j k} \int \hat{\Psi}_{j}^{+}(\vec{r}) \hat{\Psi}_{k}^{+}(\vec{r}) \hat{\Psi}_{k}(\vec{r}) \hat{\Psi}_{j}(\vec{r}) d^{3} \vec{r}, \\
H_{c}(t) & =-\frac{\hbar \Omega(t)}{2} \int\left(\hat{\Psi}_{\uparrow}^{+}(\vec{r}) \hat{\Psi}_{\downarrow}(\vec{r})+\hat{\Psi}_{\downarrow}^{+}(\vec{r}) \hat{\Psi}_{\uparrow}(\vec{r})\right) d^{3} \vec{r} .
\end{aligned}
$$

Here, $m$ is the single-atom mass, $V=\frac{1}{2} m \omega^{2} r^{2}$ is the trapping potential, $\Omega(t) \geq 0$ is the coupling strength, $\epsilon_{j}$ are the hyperfine energies, $\hat{\Psi}_{j}(\vec{r})$ are the field operators, and $U_{j k}=U_{k j}=4 \pi \hbar^{2} a_{j k} / \mathrm{m}$ are interaction strengths of 
atoms in states $|j\rangle$ and $|k\rangle$ parameterized by s-wave scattering lengths $a_{j k}$. To analyze dynamics only associated with internal degrees of freedom, we apply a single-mode approximation [14]: $\hat{\Psi}_{j}(\vec{r})=\hat{b}_{j} \phi(\vec{r})$, that is, assume that all atoms occupy the same single-particle external state $\phi(\vec{r})$. Thus the Hamiltonian is simplified as follows:

$$
\begin{aligned}
H= & -\frac{\hbar \Omega(t)}{2}\left(\hat{b}_{\uparrow}^{+} \hat{b}_{\downarrow}+\hat{b}_{\downarrow}^{+} \hat{b}_{\uparrow}\right)+G_{\uparrow \downarrow} \hat{b}_{\uparrow}^{+} \hat{b}_{\downarrow}^{+} \hat{b}_{\downarrow} \hat{b}_{\uparrow} \\
& +\sum_{j=\uparrow, \downarrow}\left(E_{0 j} \hat{b}_{j}^{+} \hat{b}_{j}+\frac{1}{2} G_{j j} \hat{b}_{j}^{+} \hat{b}_{j}^{+} \hat{b}_{j} \hat{b}_{j}\right),
\end{aligned}
$$

where $E_{0 j}=\int \phi^{*}(\vec{r})\left(-\frac{\hbar^{2} \nabla^{2}}{2 m}+V(\vec{r})+\epsilon_{j}\right) \phi(\vec{r}) d^{3} \vec{r}$ and $G_{j k}=U_{j k} \int|\phi(\vec{r})|^{4} d^{3} \vec{r}$. This simplification can successfully describe the system of uniform polarization. The Hamiltonian is equivalent to a Bose-Josephson junction [15, 16, 17], which can be realized by a BEC in a double-well trap [18]. In contrast to the double-well systems, in which negative charging energies $E_{C} \propto a_{s}$ may cause spatial collapse, the two-component systems support negative $E_{C} \propto\left(a_{\uparrow \uparrow}+a_{\downarrow \downarrow}-2 a_{\uparrow \downarrow}\right)$ with all positive $a_{j k}$.

In MF theory, by introducing variational trial states $|\Phi\rangle$ as coherent states [19], $\psi_{j}^{*}=\left\langle\Phi\left|\hat{b}_{j}^{+}\right| \Phi\right\rangle$ and $\psi_{j}=$ $\left\langle\Phi\left|\hat{b}_{j}\right| \Phi\right\rangle$ obey the classical Hamiltonian:

$$
\begin{aligned}
H_{M F}= & -\frac{\hbar \Omega(t)}{2}\left(\psi_{\uparrow}^{*} \psi_{\downarrow}+\psi_{\downarrow}^{*} \psi_{\uparrow}\right)+G_{\uparrow \downarrow}\left|\psi_{\uparrow}\right|^{2}\left|\psi_{\downarrow}\right|^{2} \\
& +\sum_{j=\uparrow, \downarrow}\left(E_{0 j}\left|\psi_{j}\right|^{2}+\frac{1}{2} G_{j j}\left|\psi_{j}\right|^{4}\right) .
\end{aligned}
$$

Rewriting $\psi_{j}=\sqrt{N_{j}} \exp \left(i \theta_{j}\right)$ in terms of the particle numbers $N_{j}$ and phases $\theta_{j}$, we denote $l=\left(N_{\uparrow}-N_{\downarrow}\right) / 2$, $L=\left(N_{\downarrow}+N_{\uparrow}\right) / 2, \Delta=E_{0 \uparrow}-E_{0 \downarrow}+L\left(G_{\uparrow \uparrow}-G_{\downarrow \downarrow}\right)$ and $E_{C}=G_{\uparrow \uparrow}+G_{\downarrow \downarrow}-2 G_{\uparrow \downarrow}$. It has been predicted that spontaneous spin polarization, a type of SSB related to self-trapping and bi-stability [15, 20], can appear when $E_{C}<0$ [7]. For symmetric systems $(\Delta=0)$ of negative $E_{C}$, the SBT occurs if $\left|\hbar \Omega / E_{C}\right|$ varies from $\left|\hbar \Omega / E_{C}\right|>L$ to $\left|\hbar \Omega / E_{C}\right|<L$. Correspondingly, the MF ground state changes from $\left(\psi_{\downarrow}, \psi_{\uparrow}\right)=$ $(\sqrt{L}, \sqrt{L}) \exp \left(i \theta_{\downarrow}\right) \quad$ to $\quad\left(\sqrt{L-l_{s}}, \sqrt{L+l_{s}}\right) \exp \left(i \theta_{\downarrow}\right)$, where $l_{s}= \pm \sqrt{L^{2}-\hbar^{2} \Omega^{2} / E_{C}^{2}}$. Below, we will focus on the SBTs in symmetric systems of $E_{C}<0$.

First, let us analyze the MF-FQ correspondence for static SBTs. It is obvious that the FQ ground state appears as an $\mathrm{SU}(2)$ coherent state if $E_{C}=0$, and the ground and first excited states become degenerate if $\Omega=0$ and $E_{C}<0$. For other arbitrary parameters, our numerical results show that the FQ ground states are always symmetric with respect to $l=0$ and the probability distributions change from single-hump shapes to double-hump ones when $\hbar \Omega / E_{C}$ passes the critical point $\hbar \Omega / E_{C}=-L$. However, the first excited states are always anti-symmetric with respect to $l=0$ and the probability distributions retain double-hump shapes.
The probability distributions and degeneracy properties of the low-energy FQ states are reminiscent of a single quantum particle confined within a potential that varies from single-well to double-well configuration. By comparing the FQ and MF ground states, it is easy to find that the MF ground states correspond to the probability maxima of the FQ ones. Because the quasi-degeneracy between the two lowest FQ states requires almost identical probability distributions for these two states, the MF bifurcation point is not identical to the degeneracy point for the two lowest FQ eigenstates. In Fig. 1, we demnstrate the MF-FQ correspondence for the static SBT in a symmetric system of total particle number $N=N_{\downarrow}+N_{\uparrow}=100$ and $E_{C}<0$. The supercritical pitchfork bifurcation of the MF stationary states signals the static SBT. Similar bifurcations have also been found in rotating BECs [21]. Due to the appearance or disappearance of unstable or stable stationary states, the bifurcation could cause dynamical instability.

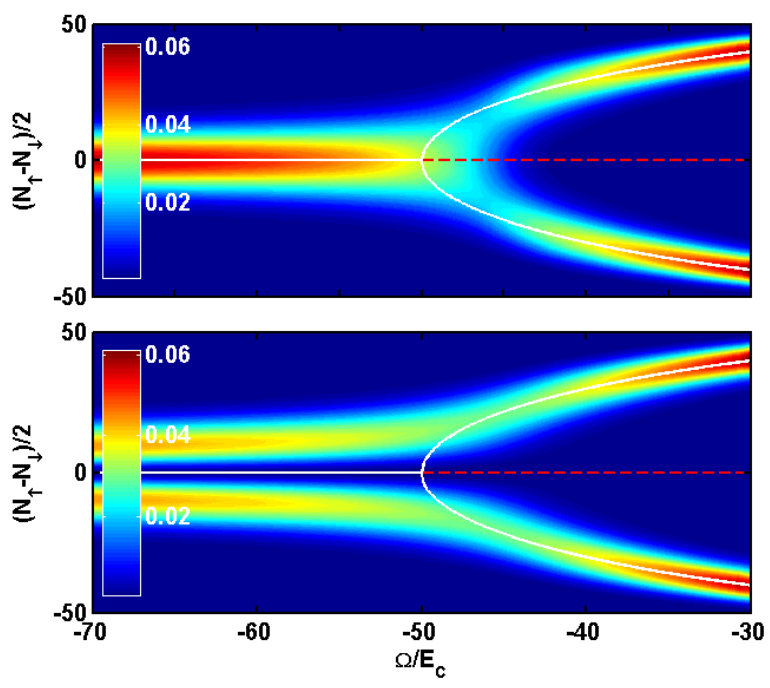

Figure 1: The MF-FQ correspondence for static SBTs in a coupled two-component condensate of $N=100$ and negative $E_{C}$. The FQ states are defined as $|\Psi\rangle=\sum_{l=-L}^{L} C(l)|l\rangle$ and the probability distributions $|C(l)|^{2}$ versus $\Omega / E_{C}$ for the ground (first-excited) states are shown in the above (bottom) panel. The solid and dashed lines are stable and unstable MF stationary states, respectively.

Now, let us consider the dynamical SBTs. In a dynamical process, there exist two characteristic time scales [4]. One time scale is the reaction time, $\tau_{r}(t)=\hbar / \Delta_{g}(t)$, which characterizes how fast the system follows eigenstates of its instantaneous Hamiltonian. Here, $\Delta_{g}(t)$ is the instantaneous excitation gap over the ground state. The other timescale is the transition time, $\tau_{t}(t)=$ $\Delta_{g}(t) /\left|d \Delta_{g}(t) / d t\right|$, which tells us how fast the system is driven. If and only if $\tau_{r}<\tau_{t}$, the system undergoes adiabatic evolution. 
To obtain the excitation modes over MF ground states, we perform a Bogoliubov analysis. Apart from a trivial gapless mode, there exists a gapped mode:

$$
\Delta_{g}=\left\{\begin{array}{l}
\hbar \sqrt{\Omega\left(\Omega-\Omega_{c}\right)}, \text { for } \Omega \geq \Omega_{c} \\
\hbar \sqrt{\Omega_{c}^{2}-\Omega^{2}}, \text { for } \Omega \leq \Omega_{c}
\end{array}\right.
$$

where $\Omega_{c}=\left|E_{C} L / \hbar\right|$. Obviously, the gap $\Delta_{g}$ gradually vanishes when $\Omega$ approaches the critical point $\Omega_{c}$.

To analyse the dynamical mechanism, we suppose $\Omega(t)=\Omega_{c}\left(1 \pm t / \tau_{q}\right)=\Omega_{c} \pm \beta t$, where $\tau_{q}$ denotes the quenching time. The relative coupling, $\varepsilon=$ $\left|\left(\Omega(t)-\Omega_{c}\right) / \Omega_{c}\right|=|t| / \tau_{q}$, corresponds to the relative temperature in KZ theory [10]. Due to the gap $\Delta_{g} \rightarrow 0$ when $\left|\Omega-\Omega_{c}\right| \rightarrow 0$, the adiabaticity breaks down when $\Omega \rightarrow \Omega_{c}$ and then revives after $\Omega$ passes $\Omega_{c}$ [see panel (a) of Fig. 2]. The time of $\tau_{r}(\hat{t})=\tau_{t}(\hat{t})$, where adiabatic-diabatic transition occurs, corresponds to the freeze-out time in $\mathrm{KZ}$ theory [10]. In the single-stable region, $\Omega>\Omega_{c}$, introducing $\Omega(t)=\Omega(t)-\Omega_{c}$, we have $\tau_{r}=\frac{1}{\sqrt{\hat{\Omega}\left(\hat{\Omega}+\Omega_{c}\right)}}$ and $\tau_{t}=\frac{2 \hat{\Omega}\left(\hat{\Omega}+\Omega_{c}\right)}{\Omega_{c}\left(2 \hat{\Omega}+\Omega_{c}\right)} \tau_{q} . \quad$ By solving $\tau_{r}(\hat{t})=\tau_{t}(\hat{t})$, we obtain the relation

$$
\tau_{q}=\frac{\left(2 \hat{\Omega}+\Omega_{c}\right) \Omega_{c}}{2\left[\hat{\Omega}\left(\hat{\Omega}+\Omega_{c}\right)\right]^{3 / 2}}=\frac{2 \varepsilon+1}{2[\varepsilon(\varepsilon+1)]^{3 / 2}} \tau_{0},
$$

where $\tau_{0}=1 / \Omega_{c}$. For slow transitions, $\tau_{q} \gg 1$, we find

$$
|\hat{t}| \approx 2^{-2 / 3} \tau_{0}^{2 / 3} \tau_{q}^{1 / 3}, \quad \varepsilon \approx 2^{-2 / 3} \tau_{0}^{-2 / 3} \tau_{q}^{-2 / 3} .
$$

In the bi-stable region, $\Omega<\Omega_{c}$, introducing $\hat{\Omega}(t)=$ $\Omega_{c}-\Omega(t)$, we have $\tau_{r}=\frac{1}{\sqrt{\hat{\Omega}\left(2 \Omega_{c}-\hat{\Omega}\right)}}$ and $\tau_{t}=$ $\frac{\hat{\Omega}\left(2 \Omega_{c}-\hat{\Omega}\right)}{\Omega_{c}\left(\Omega_{c}-\hat{\Omega}\right)} \tau_{q}$. Similarly, at the freeze-out time $\hat{t}$, we have

$$
\tau_{q}=\frac{\left(\Omega_{c}-\hat{\Omega}\right) \Omega_{c}}{\left[\hat{\Omega}\left(2 \Omega_{c}-\hat{\Omega}\right)\right]^{3 / 2}}=\frac{1-\varepsilon}{[\varepsilon(2-\varepsilon)]^{3 / 2}} \tau_{0} .
$$

For slow transitions, it is easy to find $|\hat{t}| \approx \frac{1}{2} \tau_{0}^{2 / 3} \tau_{q}^{1 / 3}$ and $\varepsilon \approx \frac{1}{2} \tau_{0}^{-2 / 3} \tau_{q}^{-2 / 3}$.

The universal scalings $|\hat{t}| \sim \tau_{0}^{2 / 3} \tau_{q}^{1 / 3}$ and $\varepsilon \sim$ $\tau_{0}^{-2 / 3} \tau_{q}^{-2 / 3}$ recover the $\mathrm{KZ}$ mechanisms: $|\hat{t}| \sim$ $\tau_{0}^{1 /(1+z \nu)} \tau_{q}^{z v /(1+z \nu)}$ and $\varepsilon \sim \tau_{0}^{-1 /(1+z \nu)} \tau_{q}^{-1 /(1+z \nu)}$ with $z=1$ and $\nu=1 / 2$, for continuous quantum phase transitions [4, 10].

Due to the adiabatic condition becoming invalid near the critical point, the defect modes could be stimulated. In Fig. 2, we show the dynamics of SBTs in the MF system. In our simulation, the initial state is chosen as a symmetry-broken ground state in the bi-stable region, and $\Omega$ is ramped up from below to above $\Omega_{c}$. For different ramping rates, we calculate the longitudinal polarization and the fidelity to instantaneous ground states. The results show that the MF defect modes appear as damped oscillations with $\beta$-dependent amplitudes [see panels (b) and (c) of Fig. 2]. The defect generation via dynamical insatiability is similar to the vortex nucleation in rotating BECs [21]. Interestingly, the defect modes become significant after the revival of adiabaticity when the system has passed the critical point, but not after the breakdown of adiabaticity when the system approaches the critical point. This indicates that the system has no sufficient time to complete a whole intrinsic oscillation in the interval between the breakdown and revival of adiabaticity. Because of this, the system tries to keep its present state when the adiabatic condition is invalid.

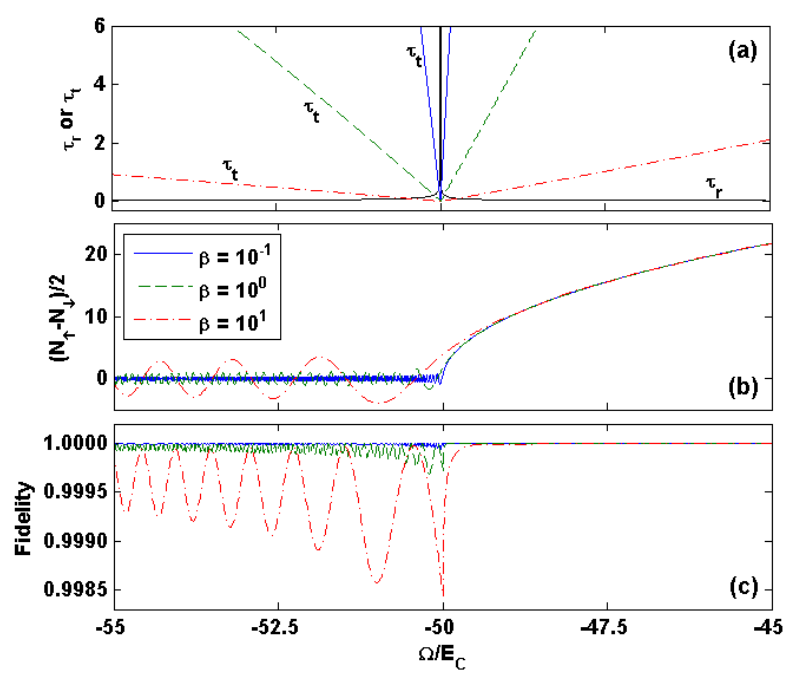

Figure 2: The MF dynamics of SBTs in Hamiltonian (2). (a) The $\tau_{t}$ and $\tau_{r}$, (b) the longitudinal polarization $\left(N_{\uparrow}-\right.$ $\left.N_{\downarrow}\right) / 2$, and (c) the fidelity to instantaneous ground states versus $\Omega / E_{C}$ for different values of $\beta$. Parameters are chosen as $N=100, G_{\downarrow \downarrow}=G_{\uparrow \uparrow}=1.0, G_{\uparrow \downarrow}=2.0$, and $E_{0 \downarrow}=E_{0 \uparrow}$. So that, $E_{C}=-2.0$ and $\Delta=0$.

To explore the correspondence between $\mathrm{MF}$ and $\mathrm{FQ}$ dynamics of SBTs, we compare the dynamics of Hamiltonians (1) and (2). In Fig. 3, we show the FQ dynamics corresponding to Fig. 2. In the FQ system, similar defect modes appear as damped oscillations. However, their critical points and oscillation regimes are different from the MF ones. First, the FQ defect modes appear after the critical point between quasi-degeneracy and non-degeneracy, which is not identical to the MF critical point between bi-stability and single-stability [see panel (b) of Fig. 3]. Additionally, the FQ defect modes have $\beta$-independent amplitudes [see panel (c) of Fig. 3]. Furthermore, starting from a ground state in the singlestable region, the MF defect modes will always appear if the system passes its critical point. However, if $|\beta|$ is sufficiently small, there is no FQ defect mode. That is, the 
FQ system always remains in its instantaneous ground state. This means that, the MF dynamics well coincides with the FQ one before the system reaches the first critical point and then it breaks down. The MF breakdown occurs at its FQ critical point if the system goes from bi-stability to single-stability. In contrat, the MF breakdown occurs at its MF critical point if the system goes from single-stability to bi-stability. This anomalous MF breakdown dependent on approaching direction differs from the conventional MF breakdown related to interaction strength and total particle number.
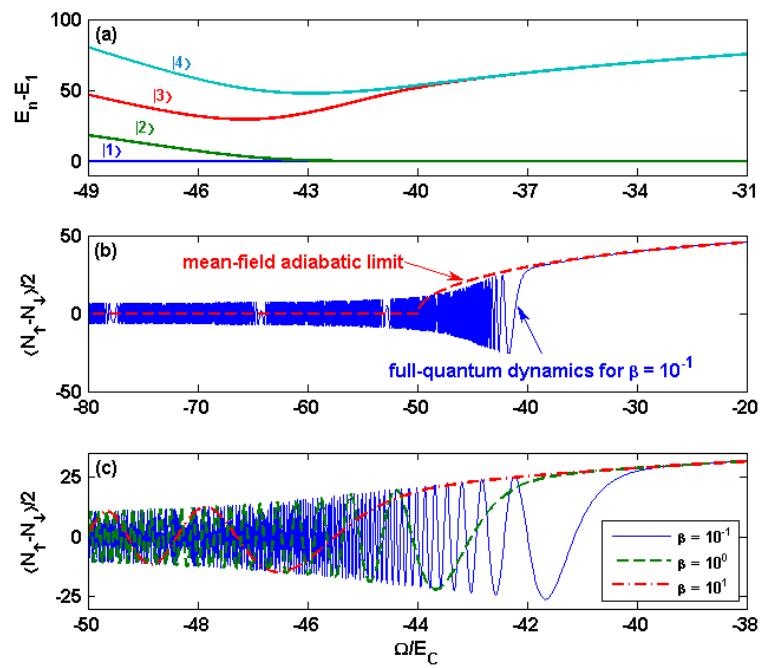

Figure 3: The energy spectrum and FQ dynamics of SBTs shown in Fig. 2. (a) Energy spectrum of four lowest states of Hamiltonian (1), (b) and (c) quantum expectations of the longitudinal polarization $\left\langle N_{\uparrow}-N_{\downarrow}\right\rangle / 2$.

To observe the dynamical SBTs, one should prepare a two-component BEC [5]. The negative $E_{C}$ with all positive s-wave scattering lengths can be obtained via Feshbach resonances. To avoid the phase separation induced by strong inter-component repulsion, one has to trap the BEC within a sufficiently strong potential. The intercomponent coupling can be realized by Raman and/or radio-frequency fields, and then the coupling strength is controlled by the field intensity. By ramping the field intensity up or down, one can push the system pass its critical points. To detect the longitudinal polarization, one should count the atoms in each component via statedependent fluorescence or spatial imaging.

In conclusion, we have studied both MF and FQ dynamics of SBTs in a coupled two-component BEC. We analytically obtain universal KZ scalings for MF dynamics and numerically explore the correspondence between MF and FQ dynamics. The MF dynamics well coincides with the FQ one before reaching the first critical point and then it breaks down. Because the FQ critical point is not identical to the MF one, the MF break- down induced by SBTs depends on the approaching direction. In a transition from the normal to polarized regions, the MF breakdown occurs at the MF critical point. However, in a transition from the polarized to normal regions, the MF breakdown occurs at the FQ critical point. This anomalous MF breakdown broadens our conventional understanding for MF breakdown related to interaction strength and total particle number.

The author acknowledges valuable discussions with Elena A. Ostrovskaya and Yuri S. Kivshar. This work is supported by the Australian Research Council (ARC).

* Electronic addresses: chl124@rsphysse.anu.edu.au; chleecn@gmail.com

[1] M. Ueda et al., AIP Conf. Proc. 869, 165 (2006).

[2] L. E. Sadler et al., Nature (London) 443, 312 (2006).

[3] H. Saito, Y. Kawaguchi, and M. Ueda, Phys. Rev. A 75, 013621 (2007); ibid. 76, 043613 (2007).

[4] B. Damski and W. H. Zurek, Phys. Rev. Lett. 99, 130402 (2007); New J. Phys. 10, 045023 (2008).

[5] J. Stenger et al., Nature (London) 396, 345 (1998); D. S. Hall et al., Phys. Rev. Lett. 81, 1539 (1998).

[6] B. D. Esry and C. H. Greene, Phys. Rev. A 59, 1457 (1999).

[7] C. Lee et al., Phys. Rev. A 69, 033611 (2004).

[8] A. Polkovnikov and V. Gritsev, Nature Phys. 4, 477 (2008); arXiv:0706.0212, arXiv:0803.3967

[9] W. H. Zurek, U. Dorner, and P. Zoller, Phys. Rev. Lett. 95, 105701 (2005); J. Dziarmaga, Phys. Rev. Lett. 95, 245701 (2005); R. W. Cherng and L. S. Levitov, Phys. Rev. A 73, 043614 (2006).

[10] T. W. B. Kibble, J. Phys. A: Math. Gen. 9, 1387 (1976); W. H. Zurek, Nature (London) 317, 505 (1985); W. H. Zurek, Phys. Rep. 276, 177 (1996).

[11] J. Dziarmaga et al., Phys. Rev. Lett. 88, 167001 (2002).

[12] R. Schützhold et al., Phys. Rev. Lett. 97, 200601 (2006); I. Klich, C. Lannert, and G. Refael, Phys. Rev. Lett. 99, 205303 (2007); J. Dziarmaga, J. Meisner, and W. H. Zurek, arXiv:0805.1025.

[13] E. Farhi et al., Science 292, 472 (2001).

[14] A. Sørensen, L. -M. Duan, J. I. Cirac, and P. Zoller, Nature (London) 409, 63 (2001); J. I. Cirac et al., Phys. Rev. A 57, 1208 (1998).

[15] S. Kohler and F. Sols, Phys. Rev. Lett. 89, 060403 (2002).

[16] C. Lee, Phys. Rev. Lett. 97, 150402 (2006); C. Lee, L. -B. Fu, and Y. S. Kivshar, EPL 81, 60006 (2008).

[17] R. Gati and M. K. Oberthaler, J. Phys. B: At. Mol. Opt. Phys. 40, R61 (2007); S. Giovanazzi et al., Phys. Rev. Lett. 84, 4521 (2000); A. Vardi and J. R. Anglin, Phys. Rev. Lett. 86, 568 (2001); D. R. Dounas-Frazer et al., Phys. Rev. Lett. 99, 200402 (2007); C. Weiss and N. Teichmann, Phys. Rev. Lett. 100, 140408 (2008).

[18] Y. Shin et al., Phys. Rev. Lett. 92, 050405 (2004); M. Albiez et al., Phys. Rev. Lett. 95, 010402 (2005); T. Schumm et al., Nature Phys. 1, 57 (2005).

[19] P. Buonsante and V. Penna, J. Phys. A: Math. Theor. 41, 175301 (2008).

[20] A. Smerzi et al., Phys. Rev. Lett. 79, 4950 (1997); G. J. Milburn et al., Phys. Rev. A 55, 4318 (1997). 
[21] S. Sinha and Y. Castin, Phys. Rev. Lett. 87, 190402 (2001). 